\title{
Development of a LAMP Method for Detecting SDHI Fungicide Resistance in Botrytis cinerea
}

F. Fan, Key Lab of Horticultural Plant Biology, Ministry of Education, and College of Plant Science and Technology; W. X. Yin and G. Q. Li, College of Plant Science and Technology and the Key Lab of Crop Disease Monitoring \& Safety Control in Hubei Province; Y. Lin and C. X. Luo, ${ }^{\dagger}$ Key Lab of Horticultural Plant Biology, Ministry of Education, and College of Plant Science and Technology, Huazhong Agricultural University, Wuhan 430070, China

\begin{abstract}
Resistance to succinate dehydrogenase inhibitors (SDHI) in Botrytis cinerea is associated with point mutations in the target gene succinate dehydrogenase subunit $B(S d h B)$. The substitution from histidine to arginine at codon $272(\mathrm{H} 272 \mathrm{R})$ is currently the predominant mutation in SDHIresistant populations in $B$. cinerea worldwide. In order to monitor the development of resistance to SDHI, a rapid, simple, and efficient method with high specificity to the H272R point mutation was developed based on loop-mediated isothermal amplification (LAMP). To specifically detect the H272R mutation, a set of four primers was designed based on the sequence of $S d h B$, and the LAMP reaction was optimized. When SYBR

Green I was added after reaction, only samples with the H272R mutation showed the color change (from brown to fluorescent yellow), indicating that this set of primers could successfully discriminate the H272R genotype from other genotypes. Specificity and accuracy tests showed that this LAMP assay had high specificity and accuracy. Moreover, the LAMP method was further simplified with fungal mycelia and conidia as the amplification template which could be prepared within $5 \mathrm{~min}$. Due to the low cost, simplicity, high efficiency, and specificity, the developed LAMP assay may contribute to the monitoring of resistance development to SDHI in B. cinerea, especially in field and high-throughput experiments.
\end{abstract}

Botrytis cinerea (teleomorph: Botryotinia fuckeliana), a fungal plant pathogen, infects over 200 crop hosts worldwide, especially vegetables and small fruit (Williamson et al. 2007). At present, control of this fungus still largely depends on fungicides. Succinate dehydrogenase inhibitors (SDHI) such as boscalid and fluopyram are among the latest fungicides most commonly used against $B$. cinerea. This class of fungicides inhibits the activity of succinate dehydrogenase $(\mathrm{SDH})$ in the mitochondrial respiratory chain (Kim and Xiao 2011). SDH, also known as mitochondrial respiratory complex II, comprises four subunits ( $\mathrm{SdhA}, \mathrm{SdhB}, \mathrm{SdhC}$, and $\mathrm{SdhD}$ ) and plays a crucial role in the tricarboxylic cycle. The inhibition of this enzyme leads to a block of the cell energy cycle, thereby resulting in cell death (Horsefield et al. 2006).

$B$. cinerea is a high-risk pathogen for the development of fungicide resistance, and its resistance to SDHI was observed only 2 years after their application in Greece, France, and Germany (Leroch et al. 2011; Veloukas et al. 2011; Walker et al. 2013). The resistance of SDHI mainly results from mutations in the target gene $S D H$. In succinate dehydrogenase subunit $B(S d h B)$, several mutations are frequently detected in resistant isolates, and have been confirmed to confer resistance to SDHI recently, such as the replacement of proline by leucine, phenylalanine, or threonine at codon $225(\mathrm{P} 225 \mathrm{~L}, \mathrm{P} 225 \mathrm{~F}$, or $\mathrm{P} 225 \mathrm{~T})$; the substitution from histidine to tyrosine, arginine, or leucine at codon 272 (H272Y, H272R, or H272L); or from asparagine to isoleucine at codon 230 (N230I) (Fernández-Ortuño et al. 2012; Lalève et al. 2014b). In addition, the substitution from histidine to arginine at codon 132 (H132R) in succinate dehydrogenase subunit $\mathrm{D}(S d h D)$ was found in field isolates with resistance to SDHI (Leroux et al. 2010). Among these mutations, H272R is presently the most common mutation associated with resistance to SDHI worldwide (De Miccolis Angelini et al. 2014; Fan et al. 2017; Fernández-Ortuño et al. 2012; Veloukas et al. 2011). The mutation H272Y or N230I was

${ }^{\dagger}$ Corresponding author: C. X. Luo; E-mail: cxluo@mail.hzau.edu.cn

Funding: This work is supported by the Special Fund for Agro-Scientific Research in the Public Interest (numbers 201303025 and 201303023).

Accepted for publication 26 February 2018.

() 2018 The American Phytopathological Society reported at lower frequencies, while $\mathrm{P} 225 \mathrm{H}$ is a rather rare mutation at present (Samaras et al. 2016). This is most likely because of a fitness cost documented recently both by isogenic and field isolates harboring N230I, P225L/F, or H272L mutations (Lalève et al. 2014a; Veloukas et al. 2014).

To manage fungicide resistance, as the first step, resistance monitoring is essential. It helps to predict the development of fungicide resistance and guide the efficient use of fungicides (Duan et al. 2014). For fungicide resistance monitoring, many methods have been established. Classic detection methods were developed using a mycelial growth inhibition and spore germination assay on media amended with different concentrations of fungicides but this procedure consumes a lot of labor and time (Duan et al. 2014). Additionally, several modern techniques were created based on single-nucleotide polymorphisms, one of the common mechanisms of fungicide resistance. For example, an allele-specific polymerase chain reaction (PCR) assay was applied to detect azoxystrobin-resistant Alternaria isolates from pistachio in California (Ma and Michailides 2004). PCR restriction fragment length polymorphism (RFLP) was used to rapidly detect benzimidazoleresistant isolates of Monilinia laxa from stone fruit and almond crops in California (Ma et al. 2005) and the demethylation inhibitor-resistant isolates of M. fructicola in the eastern United States (Luo et al. 2008). High-resolution melting (HRM) analysis was conducted to identity fenhexamid and boscalid resistance in B. cinerea (Chatzidimopoulos et al. 2014). Compared with the mycelial growth inhibition and spore germination assay, these methods are simple and time saving. However, expensive, complicated equipment and special skills are also required and, thus, these methods are not appropriate under field conditions.

Loop-mediated isothermal amplification (LAMP), a novel nucleic acid amplification method, was developed in 2000 (Notomi et al. 2000). It employs a Bst DNA polymerase and a set of four primers to amplify the target DNA with high specificity, efficiency, and rapidity under isothermal conditions (Notomi et al. 2000). In addition, it is also simple to analyze LAMP products. They can be visualized with the naked eye by adding DNA-intercalating dyes such as SYBR Green I, ethidium bromide, or Quant-iT PicoGreen (Pan et al. 2015), or by adding metal-ion indicators such as hydroxynaphthol blue (HNB), $\mathrm{CuSO}_{4}$, or calcein (Goto et al. 2009; Tomita et al. 2008; Zoheir and Allam 2011). Measuring the turbidity increase derived from magnesium pyrophosphate (a white precipitate) formation could also be adopted to visualize LAMP products (Njiru 2012). Furthermore, a real-time detection technique was also an excellent 
alternative (Dickinson 2011). Because LAMP does not require a thermal cycler, electrophoresis, or gel imaging system, it is a promising method for detection, especially in the field. To date, LAMP assays have been employed in the detection of bacteria, viruses, fungi, and transgenic plants (Kuan et al. 2016; Niessen and Vogel 2010; Wang et al. 2012; Zhou et al. 2014). Moreover, LAMP assays have also been applied in the monitoring of fungicide resistance, and herbicide resistance in weeds (Duan et al. 2014; Pan et al. 2015). However, this technology has not been used in the detection of SDHI resistance in $B$. cinerea.

In the present study, we developed a LAMP assay to rapidly detect the H272R isolates of $S d h B$ in B. cinerea. The developed LAMP detection method possesses high specificity and high sensitivity. Furthermore, the simplified LAMP method could produce results using the template directly from mycelia and conidia which could be prepared through several simple steps within $5 \mathrm{~min}$. Considering the fact that $B$. cinerea produces a lot of aerial hyphae and conidia on diseased fruit or leaves, the mycelia and conidia, harvested directly from these diseased parts, are enough for the LAMP assay. As a result, pathogen isolation is not required and the reaction could be completed in about $2 \mathrm{~h}$, indicating that the efficiency of the LAMP method was extraordinary high. Above all, the LAMP reaction doesn't require costly and intricate equipment, and can be carried out under field conditions. We believe that this new method is a rapid, simple, and efficient tool for the identification of the H272R mutation in B. cinerea, and will play an important role in SDHI fungicide resistance monitoring, especially in field and high-throughput experiments.

\section{Materials and Methods}

Fungal isolates and genomic DNA. Two isolates of $B$. cinerea, HBstr-104 and HBstr-114, were used when designing the LAMP primers.
HBstr-104 is a boscalid-sensitive isolate. Due to the H272R point mutation in gene $S d h B$, HBstr-114 is resistant to boscalid. In this study, the only other H272R-resistant isolate (HBStr-278) was analyzed along with 21 other sensitive isolates of $B$. cinerea. All of these $B$. cinerea isolates were collected from diseased greenhouse strawberry or tomato in 2012 and 2013 in Hubei Province of China, and their phenotypes to boscalid were identified in previous studies (Fan et al. 2016, 2017).

To confirm the specificity of the LAMP primers, several $B$. cinerea relative species were used, including $B$. sinoviticola, $B$. sinoallii, B. porri, Sclerotinia sclerotiorum, Monilinia fructicola, $M$. mumecola, M. yunnanensis, and Fusarium graminearum. Isolates were collected from different crops from different places. All the isolates were cultured on potato dextrose agar (PDA) plates at $22^{\circ} \mathrm{C}$ in the dark. For genomic DNA extraction, mycelia and conidia were collected using toothpick after 3 days of cultivation and the DNA was extracted and purified using the previous method (Chi et al. 2009). The final concentration of extracted DNA was about $10 \mathrm{ng} / \mu \mathrm{l}$.

Primer designing. Based on the $S d h B$ gene of $B$. cinerea, PCR primers SdhB 11F21 (CAT TCC AAG AGA CGC TAA GCA) and 760R20 (CAT CTA GAC CAC CGA CAA CA) were designed to amplify the DNA fragment containing the $\mathrm{H} 272 \mathrm{R}$ point mutation using the Oligo 7 software (Molecular Biology insights Inc.). Four specific LAMP primers were designed based on the same gene using the Primer Explorer V4 software program (http://primerexplorer.jp/ elamp4.0.0/index.html). The structure of the LAMP primers and their complementary sequence to target DNA are shown in Figure 1. The forward inner primer (FIP) consisted of the complementary sequence of F1 (F1c) and F2, and the backward inner primer (BIP) consisted of the complementary sequence of $\mathrm{B} 1$ (B1c) and B2. The outer primers $\mathrm{F} 3$ and $\mathrm{B} 3$ were essential for initiation of the reaction.

A

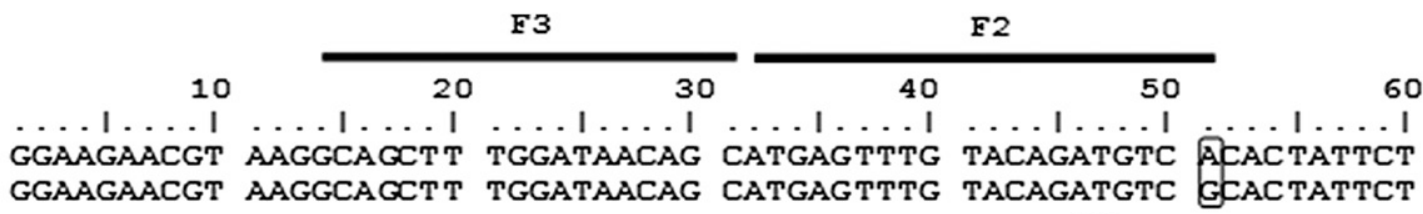

Sensitive Resistant

Sens1t1ve Resistant

Sens1tive Resistant

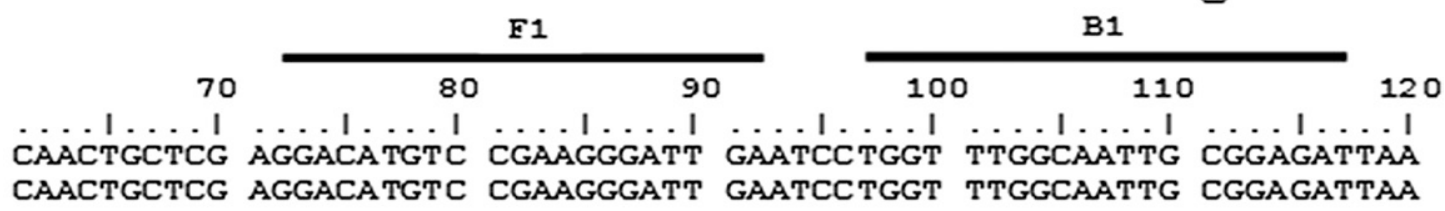

B2

\section{Sensitive Resistant

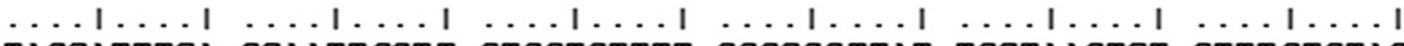 \\ TAGCATTTCA GCAATTGGTT GTGGTGTTTT GGGCGGTTAT TGCTAAGTCT GTTTGTGTAG TAGCATTTCA GCAATTGGT TTGGTGTTT GGGCGGTTAT TGCTAAGTCT GTTTGTGTAG}

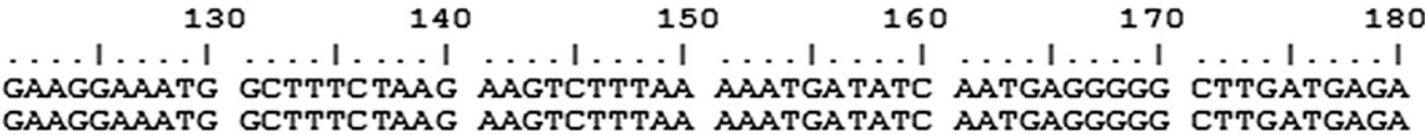

B3

$190 \quad 200 \quad 210 \quad 220 \quad 230 \quad 240$

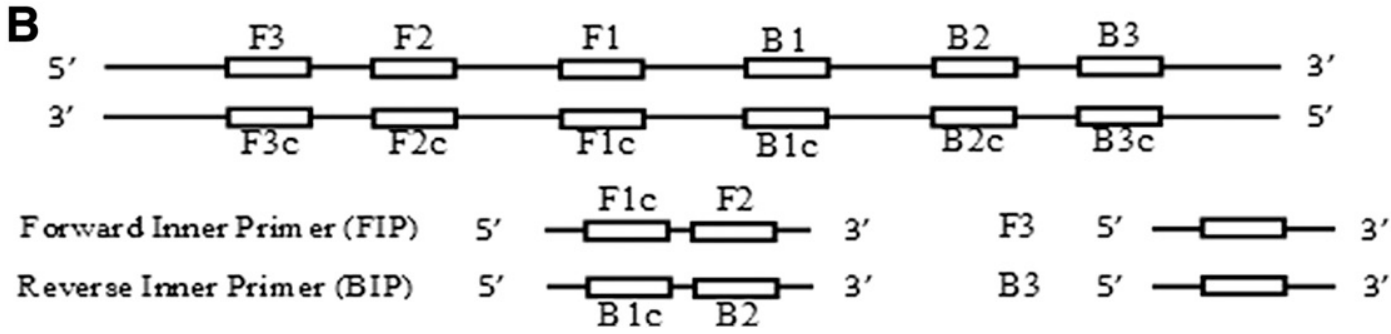

Fig. 1. Design of loop-mediated isothermal amplification (LAMP) primers for detection of the H272R mutation of SdhB in Botrytis cinerea. A, Nucleotide sequence alignment of sensitive and resistant genotypes. Sequences used as LAMP primers are indicated by bold lines. The point mutation is shown in a box. B, Schematic representation of the LAMP primers used in this work. The forward inner primer (FIP) consists of F1c and F2 and the backward inner primer (BIP) comprises B1c and B2. F1c and B1c are complementary to F1 and $\mathrm{B} 1$, respectively. 
Reaction mixture for LAMP. The initial condition of the LAMP reaction was adopted and adjusted from a former study (Duan et al. 2014). The initial LAMP reaction was carried out in a $25-\mu l$ volume containing $6 \mathrm{U}$ of Bst DNA polymerase large fragment (New England Biolabs), $2.5 \mu$ l of $10 \times$ ThermoPol Buffer (New England Biolabs), $4 \mathrm{mM} \mathrm{MgSO}_{4}$ (New England Biolabs), $1 \mathrm{mM}$ dNTP (Aidlab), $1.6 \mathrm{mM}$ each FIP and BIP, $0.2 \mathrm{mM}$ each F3 and B3, $0.94 \mathrm{M}$ betaine (Aladdin), and $10 \mathrm{ng}$ of target DNA. Pure water was used as negative control. The mixture was incubated at $63^{\circ} \mathrm{C}$ for $60 \mathrm{~min}$, then heated at $80^{\circ} \mathrm{C}$ for $10 \mathrm{~min}$ to inactivate the enzyme and terminate the reaction. The products were analyzed by $1 \%$ agarose gel electrophoresis stained with GelRed (US Everbright Inc.). Also, to visualize the reaction by the naked eye, $0.2 \mu \mathrm{l}$ of $10,000 \times$ SYBR Green I (Aidlab) was added after reaction. This experiment was repeated twice.

Optimization of LAMP reaction. The LAMP reaction was optimized in a total volume of $25 \mu \mathrm{l}$. According to previous studies (Duan et al. 2014; Zhou et al. 2014), five $\mathrm{Mg}^{2+}$ concentrations (2, 3, 4, 5, and $6 \mathrm{mM})$, four dNTP concentrations $(0.5,1,1.5$, and $2 \mathrm{mM})$, three betaine concentrations $(0,0.8$, and $1.6 \mathrm{M})$, four Bst DNA polymerase concentrations $(2,4,6$, and $8 \mathrm{U})$, four concentrations of inner primers $(0.8$, $1.2,1.6$, and $2.0 \mu \mathrm{M})$, and four concentrations of outer primers $(0.2$, $0.4,0.6$, and $0.8 \mu \mathrm{M}$ ) were tested. First, we optimized the concentration of $\mathrm{Mg}^{2+}$ while the concentrations of all the other components remained constant. Then, we optimized the concentration of dNTP at the optimal concentration of $\mathrm{Mg}^{2+}$. Accordingly, we next optimized the concentrations of betaine, Bst DNA polymerase, inner primers, and outer primers. Furthermore, to determine the optimal reaction temperature, the reaction was conducted at $61,62,63,64$, and $65^{\circ} \mathrm{C}$ for $60 \mathrm{~min}$ each. After this, the reaction was conducted at the optimal reaction temperature for $30,45,60,75$, and 90 min to determine the optimal time. The reaction was terminated by heating at $80^{\circ} \mathrm{C}$ for $10 \mathrm{~min}$. The LAMP products were examined by agarose gel electrophoresis and the color change of SYBR Green I as described before. This experiment was performed twice.

Sensitivity of LAMP and conventional PCR. The sensitivity of LAMP and PCR was assessed through performing the LAMP and conventional PCR using 10-fold serial dilutions of template DNA pMD18-SdhB, a plasmid containing the $S d h B$ gene with the H272R point mutation. To construct pMD18-SdhB, the H272R fragment was amplified by PCR with primer pair SdhB 11F21/760R20. PCR product was purified using EasyPure PCR Purification Kit (TransGen), then cloned into pMD18-T vector using the pMD18-T Vector Cloning kit (Takara). The positive clone was confirmed by both blue-white selection and sequencing. The recombined plasmid pMD18-SdhB was extracted from the culture of the positive clone using EasyPure Plasmid MiniPrep Kit (TransGen) and 10-fold serially diluted from $2 \times 10^{8}$ to $2 \times 10^{0}$ copies $/ \mu 1$.

Primer pair SdhB 11F21/760R20 was used for PCR detection. PCR was carried out in an iCycler Thermal Cycler (Bio-Rad Laboratories Inc.) in a final volume of $25 \mu \mathrm{l}$ containing $2.5 \mathrm{U}$ of EasyTaq DNA Polymerase (TransGen), $1 \times$ EasyTaq buffer, $1.25 \mathrm{mM}$ each dNTP, $0.4 \mu \mathrm{M}$ each primer, and $10 \mathrm{ng}$ of fungal template DNA. The PCR was performed with the following parameters: an initial preheating for $2 \mathrm{~min}$ at $94^{\circ} \mathrm{C}$; followed by 35 cycles of denaturation at $94^{\circ} \mathrm{C}$ for $30 \mathrm{~s}$, annealing at $58^{\circ} \mathrm{C}$ for $30 \mathrm{~s}$, and extension at $72^{\circ} \mathrm{C}$ for $60 \mathrm{~s}$; with a final extension at $72^{\circ} \mathrm{C}$ for $10 \mathrm{~min}$. The LAMP detection was completed using the optimal LAMP reaction parameters obtained from the previous section. The PCR product was checked by agarose gel electrophoresis and the LAMP products were examined by agarose gel electrophoresis and the color change of SYBR Green I. This analysis was repeated twice.

Specificity of LAMP. The specificity of LAMP was tested with genomic DNA of HBStr-114 along with several other fungal species, which were relative species of $B$. cinerea: $B$. sinoviticola, $B$. sinoallii, B. porri, S. sclerotiorum, M. fructicola, M. mumecola, M. yunnanensis, and $F$. graminearum. The negative control was performed using double-distilled water $\left(\mathrm{ddH}_{2} \mathrm{O}\right)$ instead of DNA while other reaction components remained the same. The LAMP was conducted at the optimal reaction parameters, as described before. This experiment was conducted twice.
Accuracy of LAMP. To evaluate the accuracy of LAMP, 2 known H272R isolates of B. cinerea (HBStr-114 and HBStr-278) and 21 SDHI-sensitive isolates were tested; $\mathrm{ddH}_{2} \mathrm{O}$ was used as negative control and other reaction conditions remained the same. The LAMP assay was conducted and assessed using the optimal reaction parameters, as described previously. This experiment was repeated twice.

Simplification of LAMP by using mycelia and conidia as template. In this test, two H272R isolates and five sensitive isolates were tested. To obtain mycelia and conidia, isolates were cultured on PDA plates for 7 days. The aerial mycelia and conidia on approximately $0.5 \mathrm{~cm}^{2}$ of medium were collected with toothpicks and transferred into a $1.5-\mathrm{ml}$ centrifuge tube containing $50 \mu \mathrm{l}$ of $10 \times$ Tris-EDTA (TE) buffer (100 mM Tris-HCL and $10 \mathrm{mM}$ EDTA, pH 8.0). Then, the tube was covered and boiled in water for $2 \mathrm{~min}$, followed by chilling on ice for $2 \mathrm{~min}$. Finally, it was centrifuged at 12,000 rpm for $1 \mathrm{~min}$. The supernatants obtained through these steps were used as the template. The LAMP assay was conducted and assessed at the optimal LAMP reaction parameters, as described previously. This experiment was repeated twice.

\section{Results}

Design and selection of LAMP primers. Using the Primer Explorer V4 software program, a set of LAMP primers was designed based on the sequence of gene $S d h B$ with the H272R point mutation (CAC $\rightarrow$ CGC) from boscalid-resistant isolate HBStr-114 (Fig. 1). In order to amplify the H272R mutation specifically, one nucleotide mismatch (TCG $\rightarrow \mathrm{CCG}$ ) was introduced before the mutation. This set of primers could only amplify the DNA of the resistant isolate HBStr-114, whereas nothing was amplified in the sensitive isolate HBStr-104 and the negative control (Fig. 2A). After the addition of dye, the solution of HBStr-104 and control remained brown but HBStr-114 turned to fluorescent yellow (Fig. 2A). This brown-toyellow color variance indicated the positive LAMP reaction. This set of primers could only amplify the DNA of the resistant isolate (Fig. $2 \mathrm{~B})$. These results showed that this primer set could be applied to detect the H272R mutation of $S d h B$ in B. cinerea.

Optimization of LAMP reaction. With the genomic DNA of the isolate HBStr-114, optimization of LAMP reaction was conducted. The optimal LAMP reaction was obtained in a $25-\mu l$ volume containing $4 \mathrm{U}$ of Bst DNA polymerase, $2.5 \mu \mathrm{l}$ of $10 \times$ ThermoPol Buffer, $4 \mathrm{mM} \mathrm{MgSO}_{4}, 1 \mathrm{mM}$ dNTP, $1.2 \mathrm{mM}$ each FIP and BIP, $0.4 \mathrm{mM}$ each F3 and B3, 0.8 M betaine, and $1 \mu$ l of target DNA. Furthermore, the optimal time for LAMP reaction was $60 \mathrm{~min}$ and the best temperature was $63^{\circ} \mathrm{C}$.

Sensitivity of LAMP and PCR. To test the detection limit, PCR and LAMP were performed using 10-fold serial dilutions of the plasmid pMD18-SdhB as template. PCR products were examined by agarose gel electrophoresis and LAMP products were checked by agarose gel electrophoresis and SYBR Green I. By LAMP, the ladder-like products and the color change with SYBR Green I were observed when the concentration of DNA was over $2 \times 10^{5}$ copies/ $\mu l$ (Fig. 3A and B). By PCR, a 749-bp band was amplified when the concentration of template DNA reached $2 \times 10^{3}$ copies $/ \mu$ l (Fig. $3 \mathrm{C}$ ). These results indicated that the sensitivity of LAMP was not as high as with PCR.

Specificity test of LAMP. Genomic DNA of several relative species of B. cinerea, including B. sinoviticola, B. sinoallii, B. porri, S. sclerotiorum, M. fructicola, M. mumecola, M. yunnanensis, and $F$. graminearum were used in the LAMP amplification along with B. cinerea isolate HBStr-114. The $\mathrm{ddH}_{2} \mathrm{O}$ was used as a negative control. Nothing was detected by agarose gel electrophoresis or SYBR Green I when the DNA of relative species of $B$. cinerea were treated, whereas LAMP product was acquired with the $B$. cinerea isolate HBStr-114 (Fig. 4). These results suggested that the LAMP primers had high specificity.

Accuracy of LAMP. To verify the accuracy of LAMP, 2 resistant isolates with the H272R mutation and 21 boscalid-sensitive $B$. cinerea isolates were used. As expected, only the 2 resistant isolates showed a positive reaction, whereas the reaction of the 21 sensitive isolates was negative (Fig. 5). These results demonstrated that the result of LAMP was reliable. 
LAMP amplification using DNA from boiled $B$. cinerea mycelia and conidia. To increase the time and cost efficiency of the LAMP assay, the crude DNA from mycelia and conidia was used as template to perform the LAMP amplification. The color variance of SYBR Green I occurred with mycelia and conidia of resistant isolates, whereas the sensitive ones didn't show any color change (Fig. 6A). The result was also validated by agarose gel electrophoresis. The ladder-like bands were amplified by mycelia and conidia of resistant isolates, whereas no bands were perceived from the sensitive isolates (Fig. 6B). These results show that the template obtained from mycelia and conidia through this method satisfied the requirements of LAMP.

\section{Discussion}

Gray mold, caused by B. cinerea, is a devastating disease on many crops (Williamson et al. 2007). Today, chemical control is still the principal way to control this disease. However, $B$. cinerea is a classic high-risk pathogen for the development of fungicide resistance (Brent et al. 1998). Once the resistance emerges, the efficacy of the fungicide decreases dramatically.

SDHI, registered as botryticides in recent years, are fungicides belonging to a new class with a novel mode of action. Due to their novel mode of action, SDHI do not show cross-resistance with other classes of fungicides to which the pathogen has already developed resistance (Zhang et al. 2007). Therefore, SDHI are an excellent candidate for management of fungicide resistance in B. cinerea. However, SDHI are also a fungicide of medium to high risk for resistance development, and the resistance emerged soon after its application (Fan et al. 2017; Fernández-Ortuño et al. 2012; Weber 2011). The resistance is usually conferred by several point mutations in the target gene $S d h B$ at different codons, such as P225L/F/T, H272Y/R/L, and N230I (Lalève et al. 2014b). Among all these mutations, H272R is currently
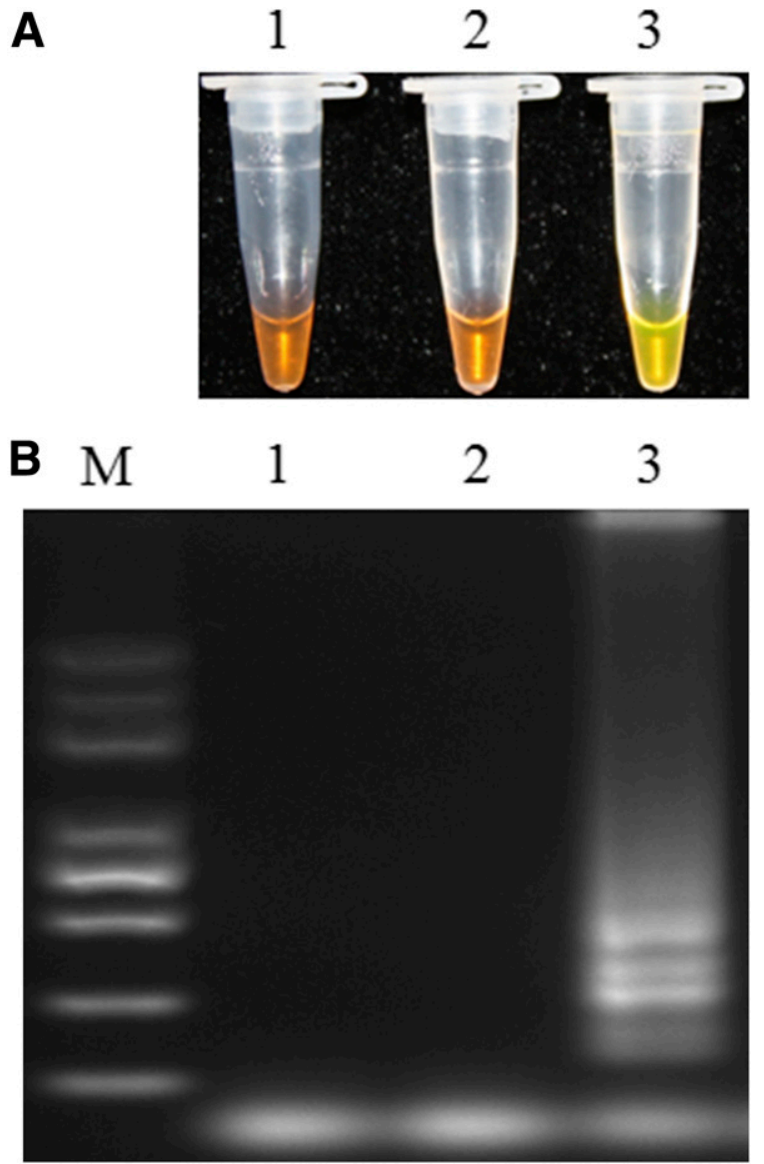

Fig. 2. Loop-mediated isothermal amplification (LAMP) for the H272R mutation of SdhB in Botrytis cinerea. A, LAMP products detected by $1,000 \times$ SYBR Green I. B, Detection of LAMP products by agarose gel electrophoresis. Lane $\mathrm{M}=\mathrm{DNA} 2 \mathrm{~K}$ plus marker, tube and lane $1=$ double-distilled $\mathrm{H}_{2} \mathrm{O}$, tube and lane $2=\mathrm{HBStr}-104$, tube and lane $3=$ HBStr-114. the predominant one in SDHI-resistant populations in $B$. cinerea around the world. It is also the genotype of resistance in Hubei Province, China (Fan et al. 2017). On the other hand, other mutations such as H272Y and N230I were reported at lower frequencies (FernándezOrtuño et al. 2012; Leroux et al. 2010; Veloukas et al. 2011). The reason for this interesting phenomenon is probably related to a fitness cost confirmed in resistant isolates with N230I, P225L/F, or H272L mutations (Lalève et al. 2014a; Veloukas et al. 2014).

To date, several techniques were established to detect and identify the mutations in $S d h B$ of B. cinerea. By target gene amplification and direct sequencing, all of the mutations could be identified. To detect the $\mathrm{H} 272 \mathrm{~L} / \mathrm{R} / \mathrm{Y}, \mathrm{P} 225 \mathrm{~F}$, and N230I mutations, a primer-introduced restriction analysis PCR assay was developed (Veloukas et al. 2011). An allele-specific PCR technique was applied for the confirmation of H272R and -Y mutations (Yin et al. 2011). For the identification of the N230I mutation, a PCR-RFLP test was conducted (Amiri et al. 2014). In order to detect and quantify N230I, P225F/L, and H272R/ Y/V mutations, a quantitative real-time allele-specific PCR was performed (De Miccolis Angelini et al. 2014). HRM analysis was adopted for the determination of $\mathrm{P} 225 \mathrm{H} / \mathrm{F} / \mathrm{L} / / \mathrm{T}, \mathrm{N} 230 \mathrm{I}$, and $\mathrm{H} 272 \mathrm{~L} / \mathrm{R} / \mathrm{Y}$ mutations (Samaras et al. 2016). However, all of these methods require expensive equipment and can be carried out only in labs. Moreover, as the first step, the isolation of the pathogens and extraction of relatively high-quality DNA are essential for most of the methods, which are time and labor consuming.

LAMP, as a novel and simple DNA amplification technique with high specificity, sensitivity, and efficiency, does not require expensive equipment, indicating that it is a promising method for detection, especially in the field (Notomi et al. 2000). In the present study, a LAMP assay was developed to detect the H272R mutation of gene $S d h B$ in B. cinerea. To our best knowledge, this is the first report that

A

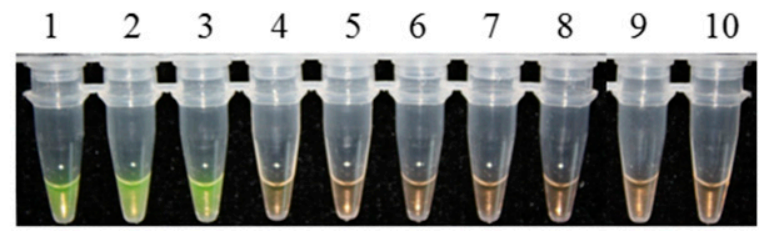

B
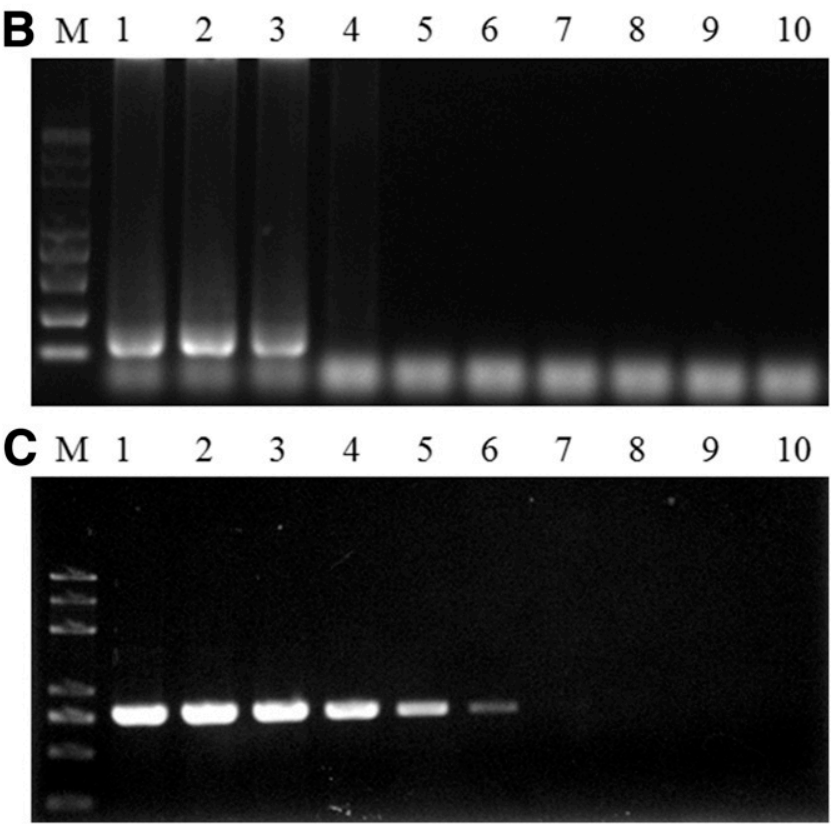

Fig. 3. Comparison of the sensitivity between loop-mediated isothermal amplification (LAMP) and conventional polymerase chain reaction (PCR). A, LAMP analysis based on 1,000x SYBR Green I. B, LAMP assessment based on agarose gel electrophoresis. C, Conventional PCR assessment based on agarose gel electrophoresis. Lane M = DNA 2K-plus marker; tubes and lanes 1 to $9=$ concentration of plasmid pMD18-sdhB at $2 \times 10^{8}, 2 \times 10^{7}, 2 \times 10^{6}, 2 \times 10^{5}, 2 \times$ $10^{4}, 2 \times 10^{3}, 2 \times 10^{2}, 2 \times 10^{1}$, and $2 \times 10^{0}$ copies/ $\mu$, respectively; tube and lane $10=$ double-distilled $\mathrm{H}_{2} \mathrm{O}$. 
LAMP was applied for the detection of fungicide resistance in B. cinerea. Up to now, LAMP has been used in the identification of bacteria, viruses, fungi, and transgenic plants (Kuan et al. 2016; Niessen and Vogel 2010; Wang et al. 2012; Zhou et al. 2014). Furthermore, a LAMP assay has also been employed in the monitoring of fungicide resistance in several plant-pathogenic fungi and herbicide resistance in weeds. In $F$. graminearum, LAMP was applied to detect the F167Y mutation of carbendazim-resistant isolates (Duan et al. 2014). In S. sclerotiorum, LAMP was adopted for the identification of benzimidazoleresistant isolates (Duan et al. 2015). Based on the LAMP method, the I1781L mutation in fenoxaprop-p-ethyl-resistant American sloughgrass could be detected (Pan et al. 2015).

According to previous studies, the sensitivity of LAMP was 100 or even 1,000 times higher than that of PCR (Duan et al. 2015; Zhou et al. 2014). Nevertheless, in our study, the sensitivity of PCR was 100 times higher than LAMP, which was contrary to previous studies. Our research revealed that LAMP could detect the template DNA at the concentration of $2 \times 10^{5}$ copies/ $\mu 1$, while other reports suggested that the detection limit of LAMP was $2 \times 10^{2}$ copies/ $\mu 1$ or $4.31 \times 10^{1}$ copies/ $\mu$ l (Duan et al. 2015; Zhou et al. 2014). For

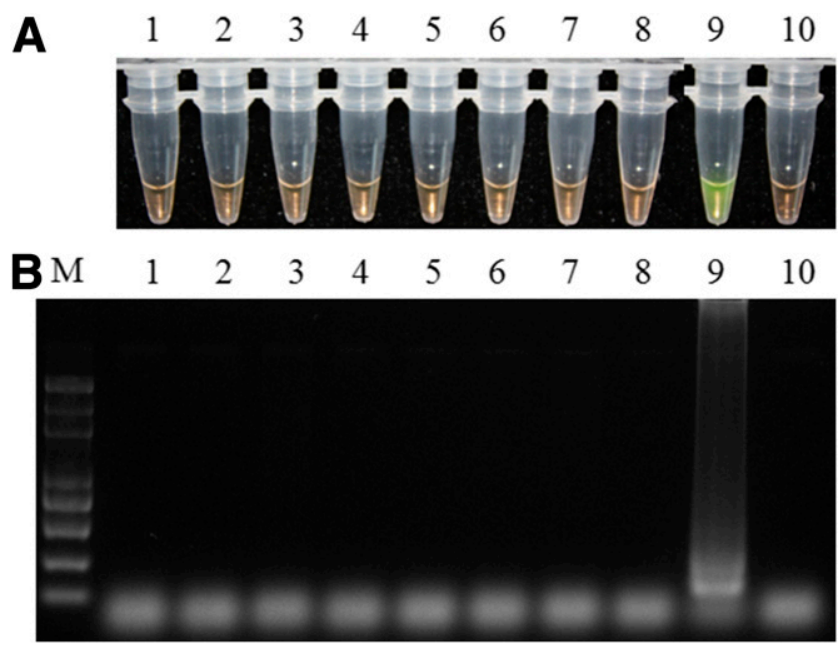

Fig. 4. Specificity of loop-mediated isothermal amplification (LAMP) detection. A, LAMP analysis based on 1,000x SYBR Green I. B, LAMP assessment based on agarose gel electrophoresis. Lane $\mathrm{M}=\mathrm{DNA} 2 \mathrm{~K}$-plus marker; tubes and lanes 1 to 8 = Botrytis sinoviticola, B. sinoallii, B. porri, Sclerotinia sclerotiorum, Monilinia fructicola, M. mumecola, M. yunnanensis, and Fusarium graminearum, respectively; tube and lane $9=B$. cinerea isolate HBStr-114; tube and lane $10=$ double-distilled $\mathrm{H}_{2} \mathrm{O}$.
PCR sensitivity, our detection limit was $2 \times 10^{3}$ copies/ $\mu$ l. This result was similar to that of a former study (Zhou et al. 2014) but was different from another previous report, which showed that the PCR detection limit was $2 \times 10^{6}$ copies/ $\mu$ l (Duan et al. 2015).Even though the sensitivity of LAMP in this study was not as high as that shown in other studies, the sensitivity was still high enough for detection.

Preparation of high-quality DNA from fungal tissue requires much time and labor, and is often a limiting step for high-throughput experiments (Chi et al. 2009). In addition, it also requires expensive and sophisticated equipment. Extraction of fungal genomic DNA generally involves two major steps: the breaking of cell walls and the extraction and purification of genomic DNA (Zhang et al. 2010). To break down cell walls, liquid nitrogen or glass rods are used in the most common methods (Zhang et al. 2010). The genomic DNA is usually extracted with cetyltrimethylammonium bromide extraction buffer (Doyle 1987) and then purified through phenol or chloroform
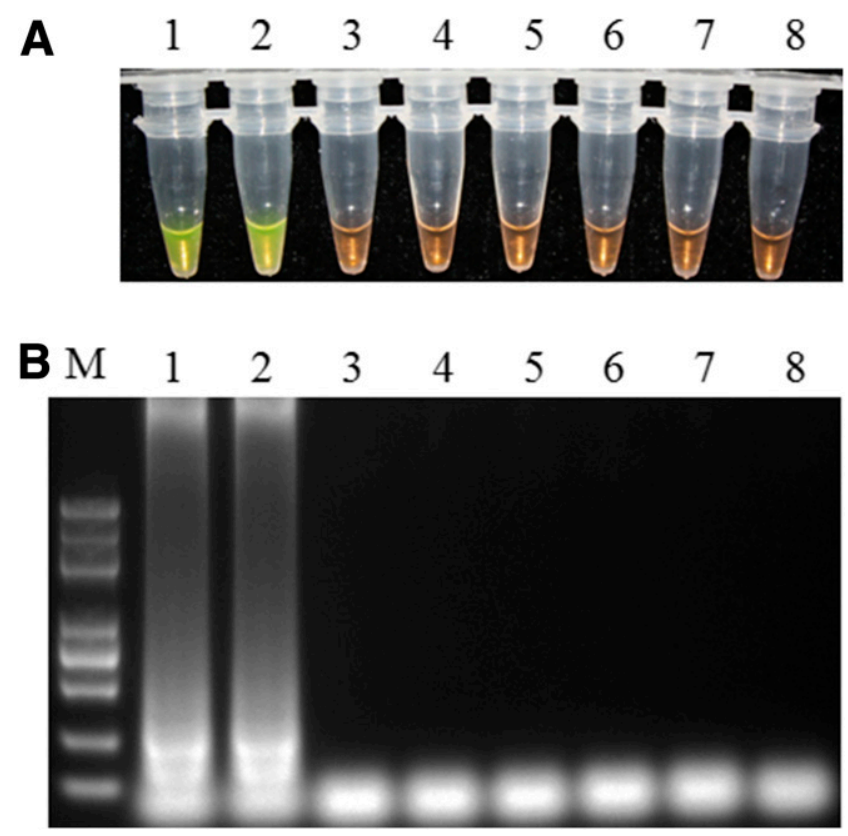

Fig. 6. Conduction of loop-mediated isothermal amplification (LAMP) detection using mycelia and conidia. A, LAMP products detected by 1,000× SYBR Green I. B, Detection of LAMP products by agarose gel electrophoresis. Lane $M=D N A 2 K-$ plus marker; tubes and lanes 1 and 2 = succinate dehydrogenase inhibitor (SDHI)resistant isolates with $\mathrm{H} 272 \mathrm{R}$ mutation; tubes and lanes 3 to $7=\mathrm{SDHI}$-sensitive isolates; tube and lane $8=$ double-distilled $\mathrm{H}_{2} \mathrm{O}$.

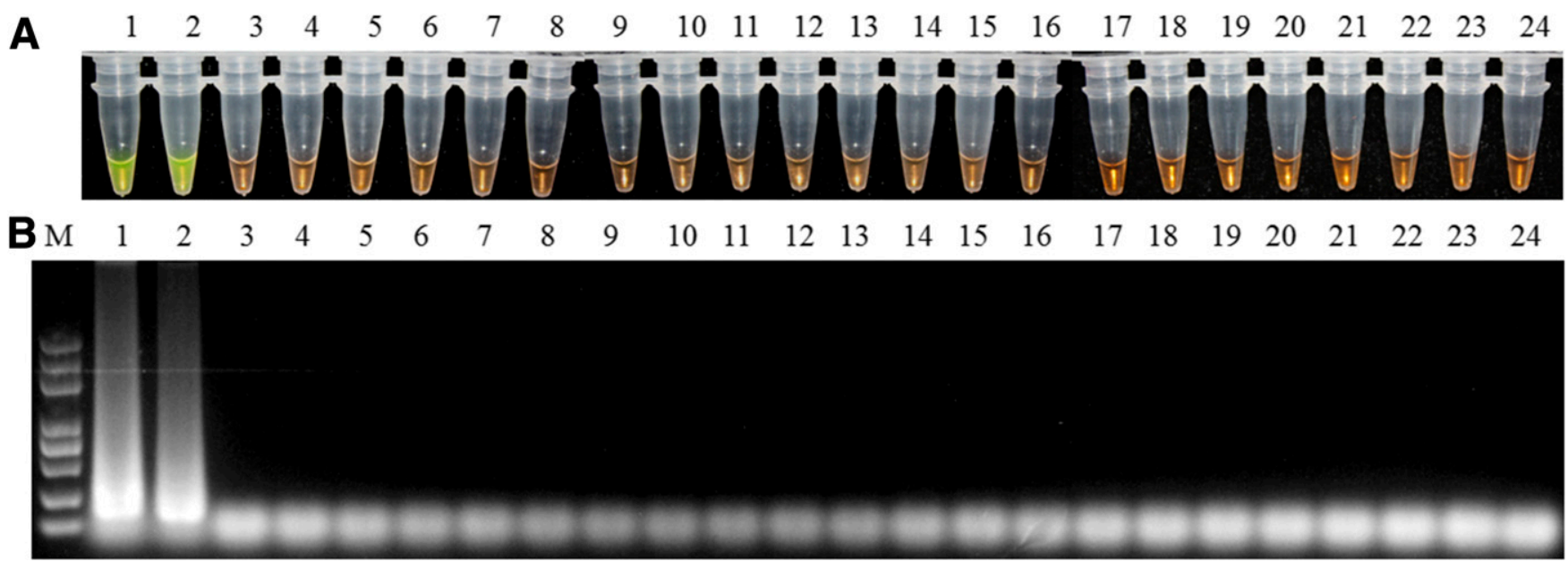

Fig. 5. Accuracy of loop-mediated isothermal amplification (LAMP) detection. A, LAMP products detected by 1,000× SYBR Green I. B, Detection of LAMP products by agarose gel electrophoresis. Lane M = DNA 2K-plus marker; tubes and lanes 1 and 2 = succinate dehydrogenase inhibitor (SDHI)-resistant isolates with H272R mutation; tubes and lanes 3 to 23 = SDHI-sensitive isolates; tube and lane $24=$ double-distilled $\mathrm{H}_{2} \mathrm{O}$. 
extraction and isopropanol or ethanol precipitation (Ashktorab and Cohen 1992). Even though DNA with satisfactory quantity and quality generally could be obtained through these techniques, these techniques are tedious and time consuming. To make DNA extraction techniques rapid and simple, abundant work has been completed, and pleasant results have been achieved (Al-Samarrai and Schmid 2000; González-Mendoza et al. 2010; Griffin et al. 2002; Zhang et al. 2010). However, these methods somewhat are still not appropriate for LAMP detection.

Based on a former study (Pan et al. 2010), a rapid and simple method was used to prepare the template DNA of LAMP amplification. By this method, template DNA is obtained from mycelia and conidia through several simple steps within $5 \mathrm{~min}$. Moreover, this method used only a little fungal mycelia. Because $B$. cinerea produces plenty of aerial hyphae and conidia on diseased fruit or leaves, it is easy to harvest the mycelia and conidia directly from the diseased fruit or leaves. This means that isolation of the pathogen is not necessary for LAMP detection, which saves a lot of labor and time. Above all, this method did not require expensive instruments or regents. To prepare the template, a centrifuge was used in this study. However, this step could be replaced by swinging the tubes roughly by hand. In addition, chilling the template on ice was optional. For reagents, only 10× TE buffer was used, which was easy to get or make with low cost.

To visualize the LAMP products, SYBR Green I and HNB are commonly used. Compared with HNB, SYBR Green I has good sensitivity and clarity (Chen et al. 2012; Zhang et al. 2013). In addition, SYBR Green I could also fluoresce in the presence of UV light. Hence, SYBR Green I is superior to other metal indicators. Because SYBR Green I inhibits the reaction, it could only be added into the mix at the end of the reaction (Goto et al. 2009). However, opening the lid to add SYBR Green I after reaction increases the chance of contamination (Lau et al. 2010). In order to solve this problem, a new closed-tube LAMP assay based on an agar dye capsule was established and showed advantages over the other closed-tube technique (Karthik et al. 2014). Moreover, SYBR Green I could be also dropped at the cap center of the tube before incubation to avoid the lid opening after reaction (Zhou et al. 2014).

The development of fungicide resistance in $B$. cinerea populations is one of the major limiting factors of successful control of this pathogen (Hahn 2014). SDHI, as one of the latest fungicides, might play a critical role in the management of fungicide resistance, because they show no cross-resistance with other classes of fungicides to which the pathogen has already developed resistance (Zhang et al. 2007). Nevertheless, SDHI are considered to be of medium to high risk for resistance development, and their resistance has been documented soon after their application, indicating that continuous monitoring of fungicide resistance is essential. The LAMP method established in the present study could be easily adopted as a simple, rapid, and efficient method to identify the resistant isolates of $B$. cinerea with $\mathrm{H} 272 \mathrm{R}$ mutation, especially if it could be achieved by using templates directly from mycelia and conidia on diseased fruit or leaves. The information obtained from resistance monitoring is a prerequisite for the successful management of fungicide resistance. Due to its low cost, high simplicity, efficiency, and specificity, the LAMP method will play a significant role in the monitoring of SDHI resistance, especially in field and high-throughput experiments.

\section{Literature Cited}

Al-Samarrai, T. H., and Schmid, J. 2000. A simple method for extraction of fungal genomic DNA. Lett. Appl. Microbiol. 30:53-56.

Amiri, A., Heath, S. M., and Peres, N. A. 2014. Resistance to fluopyram, fluxapyroxad, and penthiopyrad in Botrytis cinerea from strawberry. Plant Dis. 98:532-539.

Ashktorab, H., and Cohen, R. J. 1992. Facile isolation of genomic DNA from filamentous fungi. Biotechniques 13:198-200

Brent, K. J., Hollomon, D. W., and Federation, G. C. P. 1998. Fungicide Resistance: The Assessment of Risk. Global Crop Protection Federation, Brussels.

Chatzidimopoulos, M., Ganopoulos, I., Madesis, P., Vellios, E., Tsaftaris, A., and Pappas, A. C. 2014. High-resolution melting analysis for rapid detection and characterization of Botrytis cinerea phenotypes resistant to fenhexamid and boscalid. Plant Pathol. 63:1336-1343.

Chen, X., Wang, X., Jin, N., Zhou, Y., Huang, S., Miao, Q., Zhu, Q., and Xu, J. 2012. Endpoint visual detection of three genetically modified rice events by loop-mediated isothermal amplification. Int. J. Mol. Sci. 13:14421-14433.
Chi, M.-H., Park, S.-Y., and Lee, Y.-H. 2009. A quick and safe method for fungal DNA extraction. Plant Pathol. J. 25:108-111

De Miccolis Angelini, R. M., Masiello, M., Rotolo, C., Pollastro, S., and Faretra, F 2014. Molecular characterisation and detection of resistance to succinate dehydrogenase inhibitor fungicides in Botryotinia fuckeliana (Botrytis cinerea). Pest Manage. Sci. 70:1884-1893.

Dickinson, M. 2011. Use of a real-time LAMP isothermal assay for detecting 16 SrII and XII phytoplasmas in fruit and weeds of the Ethiopian Rift Valley. Plant Pathol. 60:1183.

Doyle, J. J. 1987. A rapid DNA isolation procedure for small quantities of fresh leaf tissue. Phytochem. Bull. 19:11-15.

Duan, Y., Zhang, X., Ge, C., Wang, Y., Cao, J., Jia, X., Wang, J., and Zhou, M. 2014. Development and application of loop-mediated isothermal amplification for detection of the F167Y mutation of carbendazim-resistant isolates in Fusarium graminearum. Sci. Rep. 4: Article 7094.

Duan, Y. B., Yang, Y., Wang, J. X., Liu, C. C., He, L. L., and Zhou, M. G. 2015. Development and application of loop-mediated isothermal amplification for detecting the highly benzimidazole-resistant isolates in Sclerotinia sclerotiorum. Sci. Rep. 5: Article 17278.

Fan, F., Hamada, M. S., Li, N., Li, G. Q., and Luo, C. X. 2017. Multiple fungicide resistance in Botrytis cinerea from greenhouse strawberries in Hubei province, China. Plant Dis. 101:601-606.

Fan, F., Li, N., Li, G. Q., and Luo, C. X. 2016. Occurrence of fungicide resistance in Botrytis cinerea from greenhouse tomato in Hubei province, China. Plan Dis. 100:2414-2421.

Fernández-Ortuño, D., Chen, F., and Schnabel, G. 2012. Resistance to pyraclostrobin and boscalid in Botrytis cinerea isolates from strawberry fields in the Carolinas. Plant Dis. 96:1198-1203.

González-Mendoza, D., Argumedo-Delira, R., Morales-Trejo, A., Pulido-Herrera A., Cervantes-Díaz, L., Grimaldo-Juarez, O., and Alarcón, A. 2010. A rapid method for isolation of total DNA from pathogenic filamentous plant fungi. Genet. Mol. Res. 9:162-166.

Goto, M., Honda, E., Ogura, A., Nomoto, A., and Hanaki, K. 2009. Colorimetric detection of loop-mediated isothermal amplification reaction by using hydroxy naphthol blue. Biotechniques 46:167-172.

Griffin, D. W., Kellogg, C. A., Peak, K. K., and Shinn, E. A. 2002. A rapid and efficient assay for extracting DNA from fungi. Lett. Appl. Microbiol. 34:210-214.

Hahn, M. 2014. The rising threat of fungicide resistance in plant pathogenic fungi: Botrytis as a case study. J. Chem. Biol. 7:133-141.

Horsefield, R., Yankovskaya, V., Sexton, G., Whittingham, W., Shiomi, K., Omura, S., Byrne, B., Cecchini, G., and Iwata, S. 2006. Structural and computational analysis of the quinone-binding site of complex II (succinateubiquinone oxidoreductase): A mechanism of electron transfer and proton conduction during ubiquinone reduction. J. Biol. Chem. 281: 7309-7316.

Karthik, K., Rathore, R., Thomas, P., Arun, T. R., Viswas, K. N., Dhama, K., and Agarwal, R. K. 2014. New closed tube loop mediated isothermal amplification assay for prevention of product cross-contamination. MethodsX 1:137-143.

Kim, Y. K., and Xiao, C. L. 2011. Stability and fitness of pyraclostrobin- and boscalid-resistant phenotypes in field isolates of Botrytis cinerea from apple. Phytopathology 101:1385-1391.

Kuan, C.-P., Lin, Y.-F., Lin, Y.-C., and Chen, C.-C. 2016. One-step reverse transcription loop-mediated isothermal amplification for the detection of Capsicum chlorosis virus in Phalaenopsis orchids. J. Gen. Plant Pathol. 82: 307-313.

Lalève, A., Fillinger, S., and Walker, A. S. 2014a. Fitness measurement reveals contrasting costs in homologous recombinant mutant of Botrytis cinerea resistant to succinate dehydrogenase inhibitors. Fungal Genet. Biol. 67: 24-36.

Lalève, A., Gamet, S., Walker, A. S., Debieu, D., Toquin, V., and Fillinger, S. 2014b. Site-directed mutagenesis of the P225, N230 and H272 residues of succinate dehydrogenase subunit B from Botrytis cinerea highlights different roles in enzyme activity and inhibitor binding. Environ. Microbiol. 16: 2253-2266.

Lau, Y. L., Meganathan, P., Sonaimuthu, P., Thiruvengadam, G., Nissapatorn, V., and Chen, Y. 2010. Specific, sensitive, and rapid diagnosis of active toxoplasmosis by a loop-mediated isothermal amplification method using blood samples from patients. J. Clin. Microbiol. 48:3698-3702.

Leroch, M., Kretschmer, M., and Hahn, M. 2011. Fungicide resistance phenotypes of Botrytis cinerea isolates from commercial vineyards in south west Germany. J. Phytopathol. 159:63-65.

Leroux, P., Gredt, M., Leroch, M., and Walker, A. S. 2010. Exploring mechanisms of resistance to respiratory inhibitors in field strains of Botrytis cinerea, the causal agent of gray mold. Appl. Environ. Microbiol. 76:6615-6630.

Luo, C.-X., Cox, K. D., Amiri, A., and Schnabel, G. 2008. Occurrence and detection of the DMI resistance-associated genetic element 'Mona' in Monilinia fructicola. Plant Dis. 92:1099-1103.

Ma, Z., and Michailides, T. J. 2004. An allele-specific PCR assay for detecting azoxystrobin-resistant Alternaria isolates from pistachio in California. J. Phytopathol. 152:118-121.

Ma, Z., Yoshimura, M. A., Holtz, B. A., and Michailides, T. J. 2005. Characterization and PCR-based detection of benzimidazole-resistant isolates of Monilinia laxa in California. Pest Manage. Sci. 61:449-457. 
Niessen, L., and Vogel, R. F. 2010. Detection of Fusarium graminearum DNA using a loop-mediated isothermal amplification (LAMP) assay. Int. J. Food Microbiol. 140:183-191.

Njiru, Z. K. 2012. Loop-mediated isothermal amplification technology: Towards point of care diagnostics. PLoS Negl. Trop. Dis. 6:e1572.

Notomi, T., Okayama, H., Masubuchi, H., Yonekawa, T., Watanabe, K., Amino, N., and Hase, T. 2000. Loop-mediated isothermal amplification of DNA. Nucleic Acids Res. 28:e63.

Pan, L., Cui, C., and Wang, B. 2010. Rapid extraction of filamentous fungal DNA for PCR amplification. Microbiol. China 37:132-135.

Pan, L., Li, J., Zhang, W. N., and Dong, L. 2015. Detection of the I1781L mutation in fenoxaprop-p-ethyl-resistant American sloughgrass (Beckmannia syzigachne Steud.), based on the loop-mediated isothermal amplification method. Pest Manage. Sci. 71:123-130.

Samaras, A., Madesis, P., and Karaoglanidis, G. S. 2016. Detection of $s d h B$ gene mutations in SDHI-resistant isolates of Botrytis cinerea using high resolution melting (HRM) analysis. Front. Microbiol. 7:1815.

Tomita, N., Mori, Y., Kanda, H., and Notomi, T. 2008. Loop-mediated isothermal amplification (LAMP) of gene sequences and simple visual detection of products. Nat. Protoc. 3:877-882.

Veloukas, T., Kalogeropoulou, P., Markoglou, A. N., and Karaoglanidis, G. S. 2014. Fitness and competitive ability of Botrytis cinerea field isolates with dual resistance to SDHI and QoI fungicides, associated with several $s d h B$ and the $c y t b$ G143A mutations. Phytopathology 104:347-356.

Veloukas, T., Leroch, M., Hahn, M., and Karaoglanidis, G. S. 2011. Detection and molecular characterization of boscalid-resistant Botrytis cinerea isolates from strawberry. Plant Dis. 95:1302-1307.
Walker, A.-S., Micoud, A., Rémuson, F., Grosman, J., Gredt, M., and Leroux, P. 2013. French vineyards provide information that opens ways for effective resistance management of Botrytis cinerea (grey mould). Pest Manage. Sci. 69:667-678.

Wang, F., Jiang, L., and Ge, B. 2012. Loop-mediated isothermal amplification assays for detecting shiga toxin-producing Escherichia coli in ground beef and human stools. J. Clin. Microbiol. 50:91-97.

Weber, R. W. S. 2011. Resistance of Botrytis cinerea to multiple fungicides in northern German small-fruit production. Plant Dis. 95:1263-1269.

Williamson, B., Tudzynski, B., Tudzynski, P., and van Kan, J. A. 2007. Botrytis cinerea: The cause of grey mould disease. Mol. Plant Pathol. 8:561-580.

Yin, Y. N., Kim, Y. K., and Xiao, C. L. 2011. Molecular characterization of boscalid resistance in field isolates of Botrytis cinerea from Apple. Phytopathology 101:986-995.

Zhang, C. Q., Yuan, S. K., Sun, H. Y., Qi, Z. Q., Zhou, M. G., and Zhu, G. N. 2007. Sensitivity of Botrytis cinerea from vegetable greenhouses to boscalid. Plant Pathol. 56:646-653.

Zhang, J., Zhu, J., Ren, H., Zhu, S., Zhao, P., Zhang, F., Lv, H., Hu, D., Hao, L., and Geng, M. 2013. Rapid visual detection of highly pathogenic streptococcus suis serotype 2 isolates by use of loop-mediated isothermal amplification. J. Clin. Microbiol. 51:3250-3256.

Zhang, Y. J., Zhang, S., Liu, X. Z., Wen, H. A., and Wang, M. 2010. A simple method of genomic DNA extraction suitable for analysis of bulk fungal strains. Lett. Appl. Microbiol. 51:114-118.

Zhou, D., Guo, J., Xu, L., Gao, S., Lin, Q., Wu, Q., Wu, L., and Que, Y. 2014 Establishment and application of a loop-mediated isothermal amplification (LAMP) system for detection of crylAc transgenic sugarcane. Sci. Rep. 4: Article 4912.

Zoheir, K. M., and Allam, A. A. 2011. A rapid improved method for sexing embryo of water buffalo. Theriogenology 76:83-87. 